I IUY.L: Luiture-specinc and pancuiturai moaeıs or approacnes to iearning: ractoriai valiaicy or diggs learning process questionnaire using confirmatory factor analysis

Darmanegara Liem, Arief National University of Singapore, Singapore

Nair, Elizabeth National University of Singapore, Singapore

B. I. Bernardo, Allan De La Salle University, the Philippines

Hidajat Prasetya, Paulus Maranatha Christian University, Indonesia

The Learning Process Questionnaire (Biggs, 1987) is a measure designed to assess students' approaches to learning (surface, deep and achieving motives and strategies) at the secondary-school level. Various theoretical models regarding to approaches to learning as operationalized by the LPQ have been noted in the literature. This study tests such models with the data sets from Singapore, Indonesia, the Philippines and Australia ( $N=230$, respectively; male and female are equally distributed). While the existing theoretical models were supported, culture-specific and pancultural model were also revealed. These findings lend the latest support to the theory and construct of approaches to learning.

1109.3: The use of unique rewards as a training tool

Woon Mok, Leh University of Minnesota, Twin Cities, USA

Bruce Overmier, J. University of Minnesota, Twin Cities, USA

Many everyday behaviors are learned and reinforced through the availability of unique rewards, e.g., when we want coffee, we go to Starbucks; but when we want pizza, we know we should go to Pizza Hut instead. The learning of this relation between the presenting stimulus (coffee), choice of where to go (Starbucks) and the outcome (getting coffee, not pizza) can be characterized as conditional discriminative choice learning. Research shows the technique of presenting unique rewards makes such learning better and faster, and has great potential to be developed into a training tool to help children and adults learn complex discriminations.

1109.4: What the hand tells the eye: The effect of copying on recognition memory Shiu, Ling-po Chinese University of Hong Kong, Hong Kong, China

Can copying a figure by hand help us recognize the figure? Participants in our experiments studied some diagrams of knots by either copying or mere looking. Then they tried to recognize those figures in both an immediate and a delayed memory test. Results show that participants who copied had better recognition memory than participants who merely looked. The benefits of copying were not found, however, if copying was done under time pressure. These results are explicable by the cognitive load theory (Sweller, Merrienboer, \& Paas, 1998). We also examine several hypotheses regarding why copying can enhance recognition memory.

\title{
1110: Cognition
}

Jump to section

\section{Convenor: Mikiya Hayashi (ORAL)}

1110.1: Emotional Stroop test for the stimuli associated with the increase and decrease of the experimental reward

Hayashi, Mikiya Nagoya University, Graduate School of Education and Human Development, Japan

This study examined the emotional Stroop interference effect for positive, negative and neutral stimuli. In the first section of the experiment, two types of stimuli were associated with the increase or decrease of the experimental reward. Another one type of stimuli was not associated with the change of reward. The amount of the increase or decrease was 10,50 , and 90 . Thus, three positively valenced stimuli and three negatively stimuli and three neutral

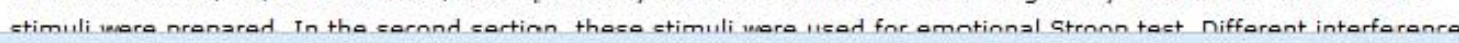

